\title{
Local anesthesia before intravenous cannula insertion: Recommendations for registered nurses in practice
}

\author{
Vera Campbell-Jones* \\ Nursing Department, Southeast Missouri State University, Cape Girardeau, MO, United States
}

Received: November 20, 2020

Accepted: January 10, 2021

Online Published: January 18, 2021

DOI: $10.5430 /$ jnep.v11n5p32

URL: https://doi.org/10.5430/jnep.v11n5p32

\begin{abstract}
Objective: The purposes of this project were to educate registered nurses of the intradermal, pretreatment procedure; provide education on the hospital's current IV therapy pretreatment policy; and increase the usage of intradermal, local anesthesia for cannulation for adult patients' comfort level.

Methods: A mixed method of nonexperimental descriptive pre- and post-survey was used. The data was collected from 48 registered nurses' pre- and post-surveys indicating descriptive analysis. The descriptive analysis identified barriers as to why registered nurses were not using pretreatment prior to IV insertion.

Results: The results revealed the majority of the participants (83\%) were not aware of the hospital's IV pretreatment policy of intradermal anesthesia with Lidocaine before IV insertion prior to the DNP project. Evidence indicated inconsistency in the use of pain management strategies during these procedures. The conclusions of this project provided an important overview of the barriers to change in clinical practice for registered nurses with IV skills.

Conclusions: An improvement project educational program, such as an educational video on how to preform intradermal pretreatment to an IV site prior to IV insertion and utilization of a manikin IV arm for simulation practice, was developed and recommended to a nursing IV therapy practice for registered nurses. Local anesthetic, such as intradermal, should become standard practice for registered nurses regarding pretreatment for pain control prior to intravenous insertion.
\end{abstract}

Key Words: Local anesthetics, Intradermal, Normal saline with preservatives (NSP), Nursing, Pretreatment, Psychometrics, Registered nurse (RN), Simulation

\section{INTRODUCTION}

Intravenous (IV) cannulation is one of the most frequently performed clinical techniques on adult patients by registered nurses (RNs) who provide IV skills. According to Bond et al., ${ }^{[1]}$ irregularity exists in the use of pain-controlling approaches during these techniques. Evidence shows that not all nurses are using pain control, even though the research for it is very positive. ${ }^{[1]}$ The design of this mixed method project was a one group pre/post-test design using content qualitative analysis to further describe themes that occurred in the data analysis of why nurses did not use pretreatment before IV insertion procedures. This DNP project was conducted to determine if local anesthetic, such as intradermal, should become standard practice for RNs regarding pretreatment for pain or discomfort control. The purpose of the methodology section of the DNP project was to use a mixed method of nonexperimental descriptive pre- and post-survey. The goal of this project will be to make education recommendations for a change in practice to include pretreatment for IV insertion via intradermal injection.

\footnotetext{
*Correspondence: Vera Campbell-Jones; Email: vjcampbelljones@ semo.edu; Address: Department of Nursing, Southeast Missouri State University, One University Plaza, MS 8300, Cape Girardeau, MO 63701, United States.
} 


\subsection{Problem of interest}

The problem of interest is that local anesthesia for cannulation is usually not offered to adult patients who are on general medical units. Peripheral venous cannulation is one of the fear and anxiety provoking procedures adult patients mention regarding everyday practice in hospitals. Most adult patients often state that this procedure causes considerable discomfort. Nurses note the reason for not providing pretreatment before IV insertion is the lack of IV therapy simulation classes to include education for RNs regarding pretreatment for pain control before insertion of IV cannula. According to Bond et al.'s ${ }^{[1]}$ research and with consideration to the constraints, several suggestions for nursing practice, education, and investigation could be made. It is imperative for nurses in practice to comprehend that for most adult patients, needles promote anxiety and may be recognized as traumatic and unpleasant. ${ }^{[2]}$ In addition, one of the top displeasure scores that adult patients report during hospitalizations is the pain felt by the insertion of IV catheters. ${ }^{[1]}$ Bond et al. ${ }^{[1]}$ indicated that less pain was reported by adult patients when IV sites were pretreated with an intradermal solution than when sites were not pretreated. However, even though this is the preferred method and included in many hospital policies, a majority of RNs are still not utilizing intradermal localization prior to IV insertion.

Peripheral venous cannulation is one of the most fearful procedures adult patients mention regarding everyday practice in hospitals. ${ }^{[1]}$ In reference to personal professional observations, most adult patients often relate that this procedure causes considerable discomfort and identify venipuncture or IV cannulation placement as a highly stressful event.

\subsection{Significance of problem of interest}

The literature has shown that IV cannulation is one of the frequent, highly intrusive techniques that RNs perform, most often daily, and regardless of what unit, floor, or department they are practicing on. ${ }^{[1]}$ Adult patients often experience discomfort or pain related to the insertion of IV for medication administration or hydration. ${ }^{[3]}$ IV cannulation is within an RN's scope of practice in the United States (U.S.). Throughout the United States, intravenous therapy policies vary from institution to institution and department to department. Additionally, literature can be linked to justification of the study. The aim of the literature review provided an underpinning and validation that guided the DNP project's methodical process. There is a much-needed improvement in RN IV therapy practices from the traditional practice of no pretreatment of IV sites prior to IV insertion to a change to pretreatment of sites with evidence-based practices (EBP) for patients who request local anesthetic prior to IV insertion. It is important

Published by Sciedu Press to note how each of the items in the literature review revealed the need to provide pretreatment to IV insertion to increase patient satisfaction and decrease pain ratings related to the common nursing practice of IV insertion. The literature review remains important to the PICOT question by revealing the need for increased nursing education regarding pretreatment prior to IV insertion and increased usage of pretreatment intradermal solutions. The PICOT questions remain as follows: Will an educational session discussing the IV cannulation policy decrease the IV cannulation knowledge deficit in RNs employed at the hospital? Will an educational session increase RN usage of pretreatment solutions?

The literature covered using one of the following solutions intradermally as a pretreatment prior to IV insertion: $1 \% \mathrm{Li}$ docaine, $2 \%$ Lidocaine, or NSP. More studies recommended Lidocaine as the solution that provided less pain when compared to NSP or NS. All of the literature reviewed agreed that patient pain ratings were higher with no pretreatment or when traditional IV nursing therapy was administrated. A change is needed in nursing IV education and practices to reach the higher standard of offering patients pretreatment of sites with intradermal solutions. A strong recommendation to educate registered nurses to change their traditional IV practices was in all of the literature reviewed and supported the DNP project and theory.

Findings of the literature review recommended that nurse practice include pretreatment of IV sites as a marker of highquality IV therapy nursing care. A compelling amount of the literature review recommendations were to educate registered nurses so as to change their traditional IV practices. One example of the recommendations registered nurses can use is a simulation-based, blended in-service education learning program regarding pretreatment prior to IV insertions. ${ }^{[4]}$

\subsection{Conceptual model}

Adult Learning theory offers the groundwork for virtual reality-based education. ${ }^{[5]}$ Knowles ${ }^{[6]}$ characterized selfdirected education as "a process in which individuals take the initiative without the help of others in diagnosing their learning needs, formulating goals, identifying human and material resources, and evaluating learning outcomes." Knowles ${ }^{[6]} \mathrm{de}-$ veloped andragogy, a conceptual framework for adult learning. According to Wang, ${ }^{[5]}$ Knowles identified six assumptions about adult learners:

1) Adults need to know why they need to learn something before undertaking effort to learn it.

2) Adults have a self-concept biased toward independent and self-directed learning.

3) Adults have acquired a great deal of life experience.

4) Adults value learning that helps them cope with the de- 
mands of their everyday life.

5) Adults are more interested in life-centered (also referred to as problem-centered or task-centered) approaches than subject-centered approaches to learning.

6) Adults are more motivated to learning by internal drives than external ones.

\subsection{PICOT question}

The components of the PICOT question are as follows:

- Population (P): RNs employed by the hospital.

- Intervention (I): An educational session discussing the hospital's IV cannulation policy.

- Comparison (C): The knowledge of the IV cannulation policy as determined by RNs' performance on pre- and posteducation surveys.

- Outcome (O): Decrease IV cannulation hospital policy knowledge deficit and increase RN usage of pretreatment solution.

- Time (T): After six months' time of data collection.

PICOT Question: Will an educational session discussing the IV cannulation policy decrease the IV cannulation knowledge deficit in RNs employed at the hospital? Will an educational session increase RN usage of pretreatment solution?

\subsection{Hypothesis}

RNs utilizing the hospital's procedures and policy for intradermal pretreatment of IV sites using NSP or $1 \%$ Lidocaine compared to not utilizing the hospital's procedures and policy for pretreatment of IV sites intradermally will result in an educational program increase in the incidence of nurses offering pretreatment and recommendations of changing $\mathrm{RN}$ IV practice after six months.

\subsection{Purpose of the project}

The DNP project's purpose was twofold. The first purpose was to determine the percentage of RNs who pretreated IV sites before cannulation and the percentage of RNs who did not pretreat IV sites before cannulation and the barriers that led to non-pretreatment. Second, to make education recommendations for a change in practice to include pretreatment for IV insertion via intradermal. It was determined after the study, a need existed to change common daily IV practices in a rural Midwest hospital in the U.S.to include a pretreatment for IV insertion as indicated by other researchers. ${ }^{[1,7-10]}$

\subsection{Sample size}

The recruitment of RN participants took place at a rural Midwest hospital in the U.S. Mixed methods data were used for this descriptive project. The sample size needed for qualitative survey research was at least 20-30; 60 for quantitative research. Therefore, this researcher needed at least 60 participants. The sample size depended on the number of RNs recruited. According to Sim, Saunders, Waterfield, and Kingstone, ${ }^{[11]}$ a sample size of at least $20-30$ RNs was needed for qualitative survey studies. According to Burmeister and Aitken, ${ }^{[12]}$ in quantitative studies for small populations (under 1,000), a sample size of $30 \%$ is needed, so approximately $60 \mathrm{RNs}$ would be needed for a population of $200 \mathrm{RNs}$. If the quantitative sample size was less than 60 on the initial recruitment, additional recruitment through other nursing units that provided IV therapy was utilized. The initial quantitative sample size was less than 60 for this DNP project; therefore, the researcher recruited additional participants through Same Day Recovery, Radiology, Pain Clinic, and the Nurse Residency nursing units. A total of 12 RNs did not complete the post-education survey. Because 48 RNs did complete the entire project (pre- and post-education surveys), a census sampling was used.

\subsection{Practice setting}

The primary practice setting for this EBP took place in a rural hospital in the Midwest of the U.S. after obtaining permission to utilize their location as a clinical practice site. The volunteer participants included RN personnel. The specific responsibility of the RNs was to care for medical and/or surgery patients on an inpatient or outpatient basis.

\subsection{Project plan activities}

The DNP project manager assumed responsibility for this DNP project's routine activities, which included reviewing the hospital's policy titled "Intravenous Therapy and Continuous Infusion of IV Fluids." Additional training included simulation by using a low-fidelity simulator, such as an intravenous insertion arm manikin. According to Munshi, Lababid, and Alyousef, ${ }^{[13]}$ level-four (SF4) low fidelity is meant to demonstrate a simple skill; for example, intradermal injections on an IV manikin arm.

The plan for the DNP project was explained clearly to ensure that RN participants followed the proper steps in proper order. $^{[14]}$ The pre-post-survey comprised questions for the recruited registered nurses who practice with IV skills who utilized the hospital's procedure/policy for pretreatment of IV sites, such as intradermal with $1 \%$ Lidocaine or NSP. The design of this study was a one group pre/post-test design using the comparison of content qualitative and quantative analysis from the pre- and post- survey responses, to further describe themes that occurred in the data analysis of why nurses did not utilize the hospital's procedure/policy for pretreatment of IV sites. 


\section{RESEARCH METHODOLOGY}

\subsection{One group pre/post-test design}

In this DNP project, at a rural Midwest hospital in the U.S., the researcher used a mixed methods nonexperimental design. The design was a one group pre/post-test design using content qualitative analysis to further describe themes that occurred in the data analysis of why nurses did not use pretreatment before IV insertion procedures. A descriptive analysis of the pre-and post-education survey responses from 48 RNs identified barriers as to why RNs were not using pretreatment prior to IV insertion. A mixture of quantitative and qualitative question types were used on the pre/post-test surveys.

\subsection{Order of design}

The researcher submitted a formal application and a research proposal for approval to the Institutional Review Committee of the hospital where the researcher conducted the project and to the Committee on Human Research and Institutional Review Board, College of Graduate and Professional Studies, at a university in the southern U.S. The DNP project manager informed each RN participant of the procedure, benefits, and risks. The participants were asked to give informed consent and were given a copy of the informed consent prior to initiation of the project. The informed consent contained the purpose of the project, rationally conceivable risks to the participants (none anticipated), explanation of the benefits of the project, alternatives to the project protocol, explanation that all participant activities are on a voluntary basis, and the contact information of the principal DNP investigator. The only exclusion criteria were nursing personnel not fluent in written and oral English communication, and non-nursing personnel, as the project pertained to IV skilled registered nurses who are employed on a medical and/or surgery floor or unit. There was no compensation paid to any participant in this project. The participants were able to withdraw at any time without penalty.

The methodology included recruitment of nurse participants and explaining the purpose of the DNP project. No patient consent was needed. Additional procedures included obtaining the informed consent of nurse participant volunteers before any review of the hospital's policy and procedure regarding IV therapy pretreatment of IV sites, showing of a short educational video demonstration of pretreatment of IV sites before IV insertion, and a low-fidelity simulation on an IV manikin arm. The nurse participants received a pre-education survey before reviewing the methodology procedure. The post-education survey was given two weeks later, before the project was concluded (approximately two to six months later).
The DNP project included the following points: how the project was implemented, the PICOT question, the project design, the participants, the procedure, how the researcher processed the data, the hypothesis development, the protocol development, the outcome analysis, how the researcher evaluated the hypothesis, and how the researcher disseminated and assured the quality of the results. The final results and data collected were disseminated in November 2019 for EBP for the IV practicing skilled nurses, nursing research, and education recommendations for change in IV therapy practice to the specific Midwest hospital in the U.S.

\subsection{Instrumentation}

After agreement for student clinical experience was granted, Institutional Review Board (IRB) approval and DNP chair endorsement, the researcher completed a literature review and created a paper pre-and post-education survey (see Tables 1 and 2). ${ }^{[15]}$ The pre- (see Table 1) and post- (see Table 2) education survey comprised questions for the recruited RNs who practiced with IV skills and who utilized the hospital's procedures and policy for pretreatment of IV sites, such as intradermal with $1 \%$ Lidocaine or NSP. The researcher compared their pre- and post-survey responses regarding if they did not utilize the hospital's procedures and policy for pretreatment of IV sites.

\subsection{Data analysis}

Descriptive statistics were calculated for RNs' years of practice, approximate number of IV insertions per week, if RNs were aware of hospital's pretreatment policy, and if RNs have ever received education on how to perform intradermal pretreatment. The qualitative statistics were analyzed to examine the differences of responses between the pre-and post-survey questions. After completion of the DNP project data collection, a professional statistician, who did not have access to any of the nurse participants' identifying personal information, was consulted to complete a mixed methods analysis. The following statistical test was used to analyze the descriptive results: a paired $t$-test assessed differences in RNs' ratings of IV insertion skill and offering of pretreatment. The chi square statistical test was used to examine the differences between the following survey questions: RN's ratings of IV insertion skill and offering of intradermal pretreatment to patients, and RN's personal experience with IV insertion for intradermal pretreatment to patients.

\subsection{Confidentiality of participants' questionnaires}

A total of 60 RNs completed the pre-education survey questionnaire. Data from the sample of RNs who completed the entire project (pre-education survey and post-education survey), ( $\mathrm{N}=48)$ was collected indicating descriptive analysis. 
Paper forms with no identifying personal information of the nurse participants were collected after informed consent was obtained. Data collection comprised paper surveys; qualita- tive using opened-end questions; and quantitative descriptive data. The documents were kept in a secured, lockable document box. No patient data was used.

Table 1. Pre-education survey/pretreatment use questionnaire

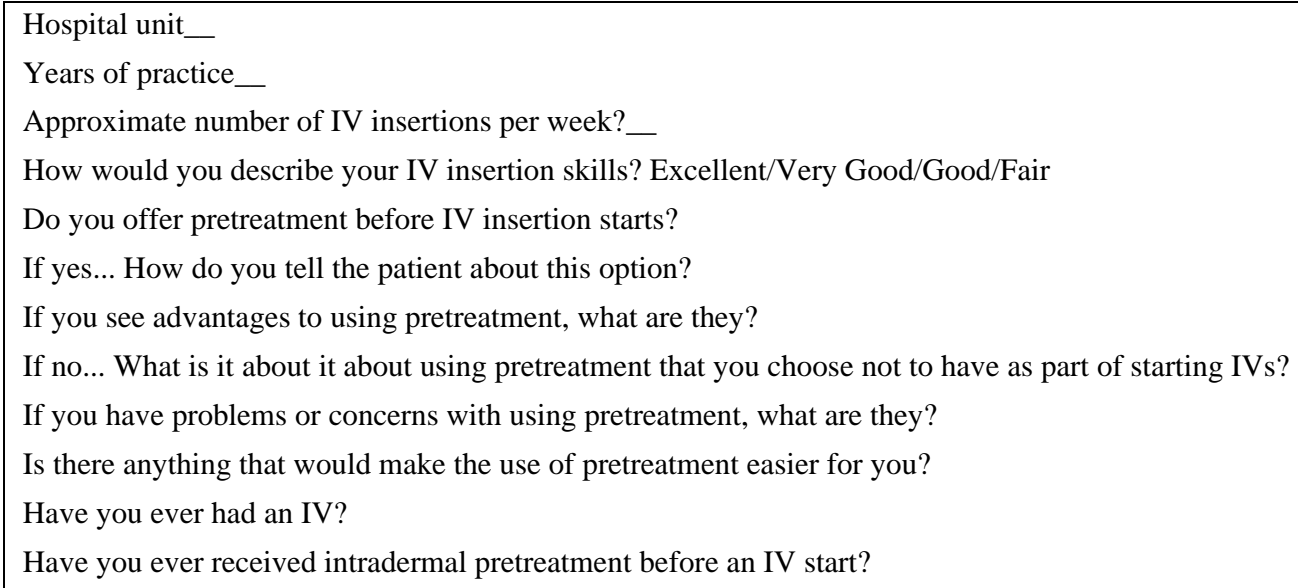

Table 2. Post-education survey/pretreatment use questionnaire

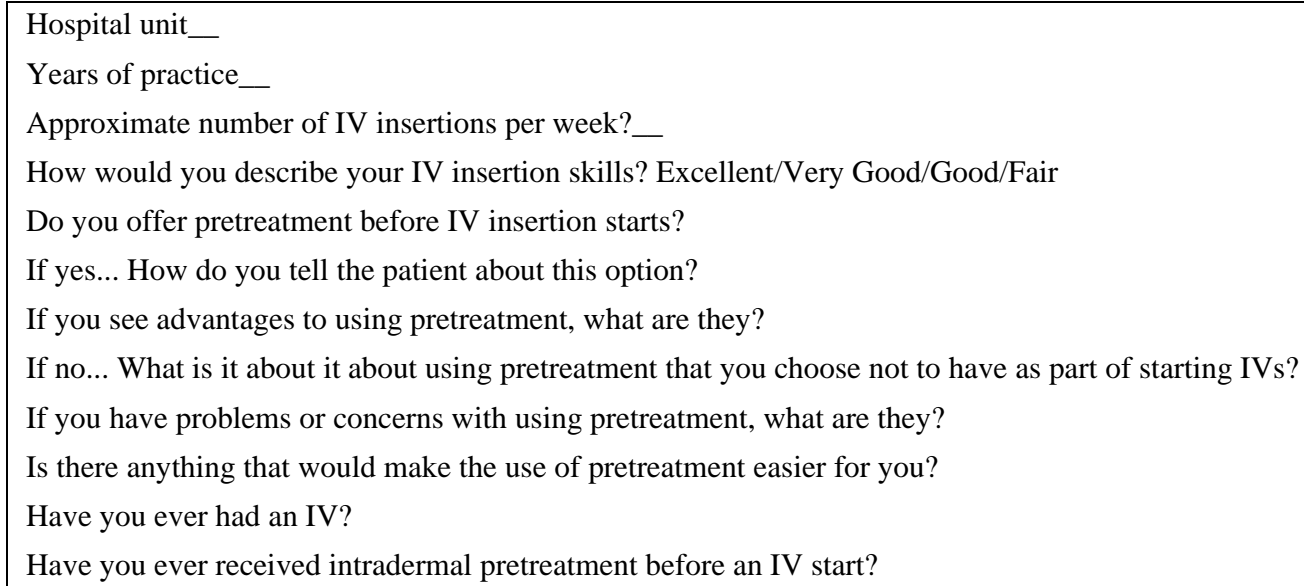

\section{FINDINGS}

\subsection{Quantitative findings}

A total of 60 RNs completed the pre-education survey. Data from the sample of RNs who completed the entire project (pre-education survey and post-education survey; $\mathrm{N}=48$ ) are reported. The data were entered into the Statistical Program for Social Services (SPSS) to facilitate answering the PICOT questions:

Will an educational session that discusses the IV cannulation policy increase knowledge of IV cannulation in RNs employed at the hospital? Will an educational session increase RN usage of pretreatment solutions?

The registered nurses had zero (or less than one year) to 40 years of IV therapy experience, with an average of 13.43 years. The approximate number of IV insertions they per- form each week ranged from one to 40, with an average of $\mathrm{n}$ $=7.25$ (pre-survey) and $n=7.896$ (post-survey). The paired $t$-test revealed: $t$-value $=0.45887, \mathrm{df}=47$, and $p$-value $=$ 6484 .

The majority of the participants were not aware of the hospital's IV pretreatment policy of intradermal anesthesia with Lidocaine before IV insertion prior to the DNP project.

Table 3. If RNs were aware of hospital's pretreatment policy

\begin{tabular}{llllll}
\hline \multirow{2}{*}{ Answer } & Post & & & Pre \\
\cline { 2 - 3 } \cline { 6 - 6 } & Number & $\%$ & & Number & \% \\
\hline No & 0 & 0.00 & & 40 & 83.33 \\
Yes & 48 & 100.00 & & 8 & 16.67 \\
Total & 48 & 100.00 & & 48 & 100.00 \\
\hline
\end{tabular}

As shown in Table 3, 83.33\% of the participants were un- 
aware of the current hospital's pretreatment policy as indicated on the pre-survey.

The percentage of registered nurses' offers regarding Lidocaine usage fell into three categories: always, sometimes, or never offered this intervention to patients. Only $10.42 \%$ of the registered nurse participants $(n=5)$ (pre-survey) stated they always offer intradermal Lidocaine before an IV insertion. The advantages they cited were that "it decreased pain at the insertion site" and "the patient appeared to experience less fear and anxiety during the IV procedure." Another $12.50 \%$ of the registered nurse participants $(n=6)$ (presurvey) stated they sometimes offer intradermal Lidocaine before an IV insertion, a variety of reasons cited, including "if the patient appeared fearful or anxious."

The majority (77.08\%) of the registered nurse participants $(\mathrm{n}=37)$ (pre-survey) stated they never offer or use intradermal Lidocaine before IV insertions. Numerous reasons were cited for not offering or not using Lidocaine as an intradermal injection for pretreatment. Many of the participants felt that "it was not reasonable to tell the patient that they would be stuck twice," or they "would use the intradermal injection pretreatment before an IV insertion if it was readily available on their unit."

\subsection{Qualitative findings}

The participants in this project explained why they were not utilizing the policy, pretreatment with intradermal anesthesia, before IV insertions.

As shown in Table 4, the number one barrier stated was, "I was not aware of this policy until you [the investigator] informed me." Other barriers listed were, "I would like clinical education on how to complete this skill"; "if the medication, Lidocaine, was readily available on the unit or convenient, I would be more inclined to use it"; "I do not want to stick my patient twice"; "because we've always started IVs without any pretreatment of intradermal injections"; and "I do not have the time."

Table 4. If RNs have ever received education on how to perform intradermal pretreatment

\begin{tabular}{llllll}
\hline \multirow{2}{*}{ Answer } & Post & & & Pre \\
\cline { 2 - 3 } \cline { 6 - 6 } \cline { 6 - 6 } & Number & $\mathbf{\%}$ & & Number & $\mathbf{\%}$ \\
\hline No & 0 & 0.00 & & 37 & 77.08 \\
Yes & 48 & 100.00 & & 11 & 22.92 \\
Total & 48 & 100.00 & & 48 & 100.00 \\
\hline
\end{tabular}

All of the barriers cited by the participants have hindered the process of implementing EBP; therefore, the hospital's current policy on IV therapy, pretreatment with intradermal injection prior to IV insertion, is not used by all partici-

Published by Sciedu Press pants with IV therapy skills. The majority of the participants voiced that administration support is needed before they can feel comfortable in utilizing the IV therapy policy. This supports the idea that ensuring an EBP environment will lead to improved outcomes for patients.

A small number of participants (10.42\%) (pre-survey) indicated that they always offered Lidocaine intradermal injection prior to IV injection and believe this procedure decreases discomfort at the site, anxiety, and fear. This number of participants $(12.50 \%)$ (post-survey) was slightly higher after education.

A slightly larger number of participants $(12.50 \%)$ (presurvey) indicated that they offered Lidocaine intradermal injection prior to IV injection only in specific circumstances, for example, if the patient appeared anxious or fearful. This number of participants (18.75\%) (post-survey) increased after the education program was given.

The remaining participants who reported not offering or using the pretreatment before IV insertion policy listed the major reason as not being aware of the current hospital policy and therefore, not offering the pretreatment for patients who asked for it. The percentage of these participants slightly decreased in never giving pretreatment after the education program, from $77.08 \%$ (pre-survey) to $68.75 \%$ (post-survey).

\section{Discussion}

\subsection{Barriers}

The descriptive analysis identified barriers as to why registered nurses were not using pretreatment prior to IV insertion. The participants listed "knowledge deficient of hospital policy" and "pretreatment medication (1\% Lidocaine, NSP) not easily accessible." The above were the top two complaints.

Change is common in the nursing profession. EBP changes are especially difficult for nurses when changing clinical practice. For a change to happen with nursing clinical practice, or for a practice to be accepted, it will have to be reasonable, effective, and convenient for nurses to make the change. ${ }^{[16]}$

According to Ginex, ${ }^{[16]}$ once a practice change is determined essential, the next phase is to integrate that evidence with clinical expertise, patient preferences, and standards. Ginex ${ }^{[16]}$ explains the last phase in EBP is to assess the outcomes and disseminate the results. The various statements from the nurses who participated in the project revealed that changing clinical practice is not easy. The DNP investigator noted that during changes in clinical practice, nurses are often faced with barriers that make change equally difficult, even with a current policy in place. 


\subsection{Limitations}

This project involved a relatively small sample of registered nurses from a rural Midwest hospital in the U.S. The chi square analysis could not be determined because some of the cells had frequencies of less than five for the following questionnaire questions: RN's ratings of IV insertion skill and offering of intradermal pretreatment to patients; and RN's personal experience with IV insertion, receiving pretreatment, and offering of intradermal pretreatment to patients.

\section{Conclusions}

\subsection{Implications for research, nursing, and analysis for leaders}

Implications for research should aim to further investigate barriers to changing IV practice and how these can be conquered; research that quantifies local anesthetic, such as intradermal injections, should become standard practice for RNs in pretreatment for pain or discomfort control. Implications for nursing, analysis for leaders and recommendations include a policy change from traditional pretreatment (no pretreatment) to intradermal pretreatment offered to patients requiring IV insertions; the addition of intradermal pretreatment policies for hospitals which do not presently have a policy in place; the compliance of registered nurses with current hospital intradermal pretreatment policies; the addition of hospital inservice educational and training programs to develop the skills registered nurses need to be successful in performing intradermal pretreatment prior to IV insertion; the usage of an educational tool-simulation or low-fidelity, for example IV manikin arm activities to enhance IV intra- dermal pretreatment skills; and nursing administrative and managerial support and encouragement for hospital registered nurses to perform intradermal pretreatment before IV insertion procedures.

\subsection{Recommendations for registered nurses in practice}

This study provided an important overview of the barriers identified to make education recommendations for a change in clinical practice to include pretreatment for IV insertion via intradermal injection for RNs with IV skills. The major changes needed are the enforcement of the hospital's policy and encouragement from administration. To facilitate the education process of the hospital's IV policy, the participants will be required to attend mini in-services regarding the clinical skill set to successfully perform intradermal injections and complete competency demonstration of this procedure. Once support by RNs providing intradermal pretreatment and by the hospital's nursing administration and managerial staff has been established, the IV skill of performing intradermal pretreatment on all adult units could be implemented at the rural Midwest hospital in the U.S. Intradermal pretreatment policies could be enforced to include new educational and training programs for all IV-skilled practicing RNs.

\section{ACKNOWLEDGEMENTS}

I would like to express my deepest appreciation to all of the nurse participants from the Midwest hospital in the U.S. who helped with the achievement of my research.

\section{CONFlicts OF INTEREST Disclosure}

The author declares that there is no conflict of interest.

\section{REFERENCES}

[1] Bond M, Crathorne L, Peters J, et al. First do no harm: Pain relief for the peripheral venous cannulation of adults, a systematic review and network meta-analysis. BMC Anesthesiology. 2016; 16: 1-11. PMid:27716082 https://doi.org/10.1186/s12871-016-025 2-8

[2] Mclenon J, Rogers MM. The fear of needles: A systematic review and meta-analysis. J of Advanced Nursing. 2019 Jan; 75(1): 30-42. PMid:30109720 https ://doi .org/10.1111/jan.13818

[3] Keleekai NL, Schuster CA, Murray CL, et al. Improving nurses' peripheral intravenous catheter insertion knowledge, confidence, and skills using a simulation-based blended learning program. Journal of Society for Simulation in Healthcare. 2016 Dec; 11(6): 376-384 PMid:27504890 https : //doi.org/10.1097/sih00000000000 00186

[4] Garner S, Prater L, Raj L, et al. Effectiveness of peripheral intravenous skill continuing education using low-fidelity simulation among nurses in India. The Journal of Continuing Education in Nurs- ing. 2018; 49(6): 255-261. PMid:29847683 https ://doi.org/10 .3928/00220124-20180517-05

[5] Wang EE. Simulation and adult learning. DM. 2011 Nov; 57(11): 664-678. PMid:22082552 https://doi.org/10.1016/j.disa month. 2011.08.017

[6] Knowles MS. Self-directed learning: a guide for learners and teachers. New York: Cambridge Books; 1975. 135 p.

[7] Dwyer V, Rutkowski B. Intradermal lidocaine for peripheral IV insertion: a change in practice. Journal of PeriAnthesia Nursing. 2013; 28(3): 49-50. https://doi.org/10.1016/j.jopan. 2013 .04 .141

[8] Levitt F, Ziemba-Davis M. An exploratory study of patient preferences for pain management during intravenous insertion: maybe we should sweat the small stuff. Journal of PeriAnesthesia Nursing. 2013; 28(4): 223-232. PMid:23886287 https://doi.org/10.1 016/j.jopan.2012.11.008

[9] Oman KS, Fink R, Kleiner C, et al. Intradermal lidocaine or bacteriostatic normal saline to decrease pain before intravenous catheter insertion: a meta-analysis. Journal of PeriAnesthesia Nursing. 2014; 
29(5): 367-376. PMid:25261140 https://doi, org/10.1016/j. jopan.2013.12.008

[10] Santana P. Use of subcutaneous local anaesthetic in venous catheters channelling for reducing pain. International Journal of Nursing. 2015; 2(2): 179-186. https://doi.org/10.15640/ijn.v2n2a18

[11] Sim J, Saunders B, Waterfield J, et al. Can sample size in qualitative research be determined a priori? International Journal of Social Research Method. 2018; 21(5): 619-634. https ://doi.org/10.108 $0 / 13645579.2018 .1454643$

[12] Burmeister E, Aitken L. Sample size: how many is enough? Australian Critical Care. 2012; 25(4): 271-274. PMid:22835279 https: //doi.org/10.1016/j.aucc.2012.07.002

[13] Munshi F, Lababid H, Alyousef S. Low- versus high-fidelity simulations in teaching and assessing clinical skills. Journal of Taibah University Medical Sciences. 2015; 10(1): 12-15. https://doi.or $\mathrm{g} / 10.1016 / \mathrm{j} \cdot \mathrm{jtumed} .2015 .01 .008$
[14] Adu P. Conducting qualitative research: the methodology chapter [Slideshare presentation]. Chicago: The Chicago School for Professional Psychology; 2016 February 3; 31 slides. Available from: https://www.slideshare.net/kontorphilip/writin g-the-methodology-chapter-of-a-qualitative-study

[15] Brown J. Registered nurses' choices regarding the use of intradermal lidocaine for intravenous insertions: the challenge of changing practice. The Journal of Pain Management Nursing. 2002; 3(2): 71-76. PMid:12050838 https://doi.org/10.1053/jpmn.2002.1230 15

[16] ONS Voice [Internet] Pittsburgh (PA): Oncological Nurses Association; c2020. Ginex PK. Overcome barriers to applying an evidence-based process for practice change; 2018. Available from: https://voice.ons.org/news-and-views/overcome-bar riers-to-applying-an-evidence-based-process-for-p ractice-change 